\title{
Randomised controlled trial of postnatal sodium supplementation on oxygen dependency and body weight in 25-30 week gestational age infants
}

\author{
Gary Hartnoll, Pierre Bétrémieux, Neena Modi
}

\begin{abstract}
Aim-To compare the effects of early against delayed sodium supplementation on oxygen dependency and body weight, in preterm infants of 25-30 weeks of gestational age.

Methods-Infants were stratified by gender and gestation and randomly assigned to receive a sodium intake of $4 \mathrm{mmol} / \mathrm{kg} /$ day starting on either the second day after birth or when weight loss of $6 \%$ of birthweight was achieved. Daily sodium intake, serum sodium concentration, total fluid intake, energy intake, clinical risk index for babies (CRIB) score and duration of ventilatory support and additional oxygen therapy were recorded. Infants were weighed daily. Weights at 36 weeks and six months of postmenstrual age were also recorded.
\end{abstract}

Results-Twenty four infants received early, and 22 delayed, sodium supplementation. There were no significant differences in total fluid and energy intake between the two groups. There was a significant difference in oxygen requirement at the end of the first week, with $9 \%$ of the early group in air in contrast to $35 \%$ of the delayed group (difference $26 \%, 95 \%$ confidence interval 2, 50). At 28 days after birth the proportions were $18 \%$ of the early group and $40 \%$ of the delayed group (difference 22\%, 95\% CI -5, 49). Proportional hazards modelling showed early sodium supplementation and lower birthweight to be significantly associated with increased risk of continuing oxygen requirement. The delayed sodium group had a greater maximum weight loss (delayed $16.1 \%$; early $11.4 \%, p=0.02$ ), but there were no significant differences in time to maximum weight loss, time to regain birthweight, and weight at 36 weeks and 6 months of postmenstrual age.

Conclusion-In infants below 30 weeks of gestation, delaying sodium supplementation until at least $6 \%$ of birthweight is lost has a beneficial effect on the risk of continuing oxygen requirement and does not compromise growth.

(Arch Dis Child Fetal Neonatal Ed 2000;82:F19-F23)

Keywords: sodium intake; postnatal weight loss; chronic lung disease; growth

Sodium is a growth promoting factor and adequate sodium supplementation is necessary for optimal growth and development after preterm birth. ${ }^{1}$ However, the time at which sodium supplementation should begin is unclear. Loss of interstitial fluid from the extracellular compartment is a prerequisite for successful postnatal adaptation and is indiccated clinically by weight loss. ${ }^{2}$ As sodium is the principal electrolyte in extracellular fluid, negative sodium balance is the physiological norm immediately after birth. ${ }^{2}$ The timing of the loss of extracellular fluid is closely linked to cardiopulmonary adaptation and, for example, is delayed in infants with respiratory distress syndrome. ${ }^{3}$ In addition, neonates, especially if preterm, have a limited ability to excrete a sodium load. ${ }^{4}$ There are therefore justifiable concerns that early sodium supplementation, especially in preterm infants with respiratory distress syndrome, will favour persistent expansion of the extracellular compartment and retention of interstitial fluid. This in turn would explain the repeated clinical observation linking failure to lose weight after birth with increased risk of respiratory morbidity, ${ }^{5}$ symptomatic patent ductus arteriosus, ${ }^{67}$ and necrotising enterocolitis. ${ }^{8}$

It is also the case that renal conservation of sodium is immature in preterm infants below 32 weeks of gestation. Impaired reabsorption at both proximal and distal tubules results in renal salt wasting. ${ }^{9}$ Intestinal absorption is also limited. ${ }^{10}$ It is therefore now standard practice in neonatal intensive care to provide a sodium intake of $4-6 \mathrm{mmol} / \mathrm{kg} / \mathrm{day},{ }^{11}$ and in many institutions this is routinely started on the second or third day after birth, regardless of clinical status.

This randomised, controlled trial aimed to determine the optimal time at which to start routine sodium supplementation after preterm birth. The null hypothesis tested was that the timing of sodium supplementation in preterm neonates with respiratory distress syndrome does not affect the risk of continuing oxygen dependency.

\section{Methods}

Infants born between 25 and 30 weeks of gestation, admitted to the neonatal intensive care units at the Hammersmith and Queen Charlotte's Hospitals, London, and requiring respiratory support (either continuous positive airways pressure (CPAP) or positive pressure ventilation) for respiratory distress syndrome, were eligible for entry. Infants with major congenital malformations, chromosomal, or renal abnormalities were excluded. The trial was 
approved by the institutional research ethics committee. Informed parental consent was obtained.

Infants were randomly allocated to receive early or delayed sodium. Randomisation was computer generated, stratified by gender and gestational age. If allocated to the early limb of the trial, the infant was not prescribed sodium for the first 24 hours after birth, but $4 \mathrm{mmol} /$ $\mathrm{kg} /$ day thereafter. Infants in the delayed limb of the trial were prescribed $4 \mathrm{mmol} / \mathrm{kg} /$ day only when $6 \%$ of birthweight had been lost. This choice was based on our previous work showing that $6 \%$ was representative of postnatal weight loss in healthy preterm infants. ${ }^{12}$ Clinical staff caring for the infants were blinded to which limb of the trial the baby was allocated. The appropriate amount of sodium was administered in parenteral nutrition fluid by pharmacy staff. Total fluid volumes were prescribed by attending clinical staff. Hyponatraemia was managed by a reduction in total fluid volume and hypernatraemia by an increase. Glucose flow rate was kept constant by altering the proportions of $5 \%$ and $50 \%$ dextrose infused. ${ }^{13}$ Parenteral energy intakes were not altered. Infants were initially nursed on radiant warmer beds with heat shields until stable and then transferred to humidified incubators.

Birthweight, gestational age, surfactant administration and maternal details, including exposure to antenatal steroids, were recorded. Infants were weighed daily using scales accurate to $\pm 10 \mathrm{~g}$. Weights were recorded until birthweight was regained, or for one week, whichever was the longer period. Total daily fluid, sodium, and energy intakes were calculated from medical and nursing records. Recorded fluid intake included all crystalloid, colloid, drug delivery and flush volumes. True sodium intake was calculated from the sodium concentration and volume of fluid administered. Serum sodium values were recorded daily for the first week, then weekly for a further two weeks. CRIB scores ${ }^{14}$ were calculated for each infant at 12 hours. Weights were also recorded at 36 weeks and 6 months of postmenstrual age.

Duration of ventilator and CPAP support were recorded, in addition to total length of time each infant received supplemental oxygen. Oxygen status was recorded at 7 and 28 days after delivery and at 36 weeks of postmenstrual age. In our neonatal units infants are

Table 1 Study infants

\begin{tabular}{lll}
\hline & Early $(n=24)$ & Delayed $(n=22)$ \\
\hline Male:female & $14: 10$ & $12: 10$ \\
Gestation (weeks/days) & $28 / 0(26 / 0,30 / 0)$ & $27 / 3(25 / 5,29 / 6)$ \\
Birthweight $(\mathrm{g})$ & $945(745,1560)$ & $946(420,1570)$ \\
Antenatal steroids & 23 & 22 \\
Antenatal thyrotropin releasing hormone & 12 & 14 \\
Delivery by caesarean section & 16 & 13 \\
CRIB score & $2(1,10)$ & $3.5(0,16)$ \\
Surfactant administration & 21 & 21 \\
Total fluid intake in first week $(\mathrm{ml} / \mathrm{kg})$ & $1084(804,1306)$ & $1087(740,1377)$ \\
Total energy intake in first week $(\mathrm{kcal} / \mathrm{kg})$ & $392(302,579)$ & $461(257,617)$ \\
a:A ratio day 1 & $0.40(0.12,0.72)$ & $0.39(0.10,0.70)$ \\
Ventilation (days) & $3(0,68)$ & $3(0,40)$ \\
\hline
\end{tabular}

Values are total or median (range). Differences are all non-significant $(p>0.05)$. regarded as no longer requiring additional oxygen when oxygen saturation in air is consistently above $96 \%$. Any occurrence of a clinically significant patent ductus arteriosus, defined as an echocardiographically confirmed patent duct giving rise to clinical signs, and necrotising enterocolitis, defined as abdominal tenderness, distension, and radiological evidence of intramural gas, was documented.

Data were tested for normality and analysed using parametric or non-parametric tests, as appropriate. Summary measures were derived to compare repeated measures. Initially, $\chi^{2}$ analysis was used to explore oxygen dependency in the two randomised groups at 7 and 28 days after delivery and at 36 weeks of postmenstrual age. Factors associated with increasing duration of additional oxygen were assessed using the Cox proportional hazards model. The assumption of proportional hazards was tested by calculating the Cox regression model, using a single variable, and comparing the probability estimate obtained from that calculation with the probability estimate obtained from the Gehan generalisation of the Wilcoxon test for the equality of two survival curves, which does not assume proportional hazards. Continuous data were divided into two categories to perform the Wilcoxon test. A graph of $\log (-\log (\mathrm{S}))$ vs $\log$ (time to no longer requiring oxygen) was then plotted for each of the variables, where $S$ is the Kaplan-Meier survival estimate. If the two probability estimates obtained from the Cox regression and the Wilcoxon test are similar and the plots of the logs of the cumulative hazards are parallel, then the assumption of proportional hazards is valid. After this analysis there was no evidence to contradict the assumption of proportional hazards. All statistical calculations were carried out using Intercooled Stata (version 4.0, Stata Corporation; Texas, USA).

\section{Results}

Twenty four infants (14 boys, 10 girls) were recruited into the early and 22 (12 boys, 10 girls) into the delayed sodium group. Details of the infants recruited to the study are shown in table 1 and daily fluid and energy intakes in figs 1 and 2. There were no significant differences between the groups. No infant received diuretics or pancuronium the first week after birth. There were three deaths in each group by 36 weeks of postmenstrual age.

Although not formally prescribed on the first day after birth, both groups received a substan-

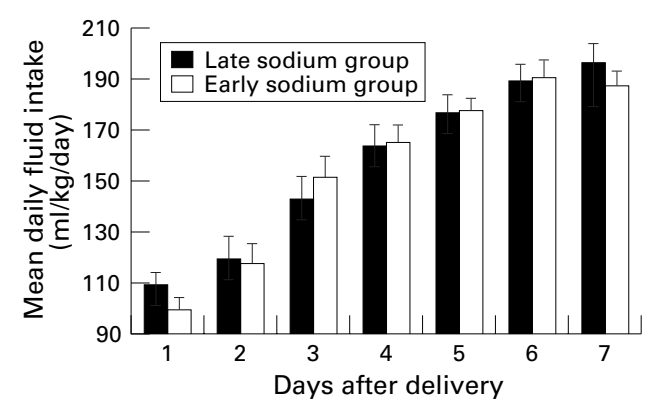

Figure 1 Daily fluid intake ( $\mathrm{ml} / \mathrm{kg} /$ day): values are mean (SEM). 


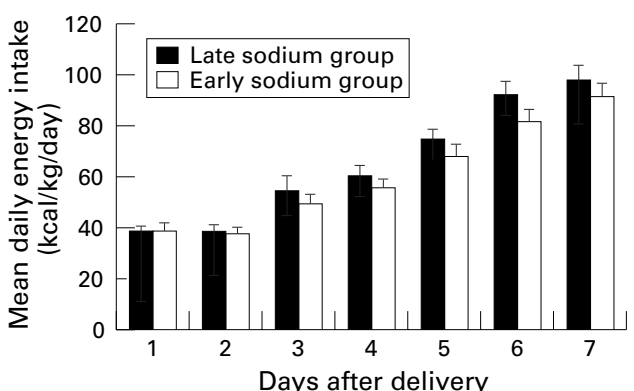

Figure 2 Daily energy intake (kcal/kg/day): values are mean (SEM).

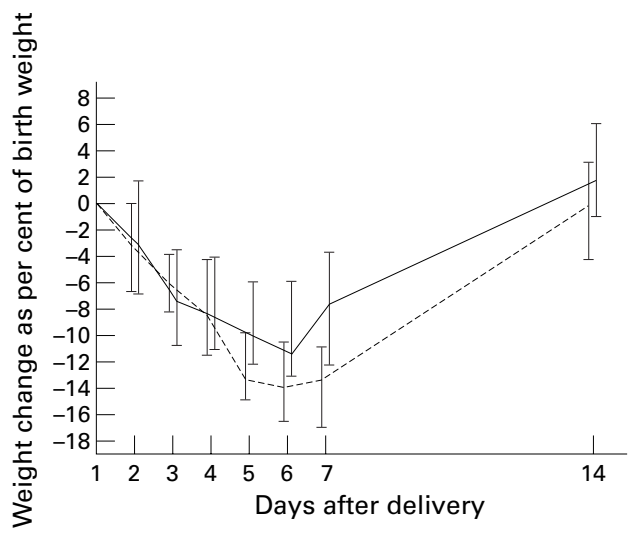

Figure 3 Body weight as a percentage of birthweight, days 1 to 14. Broken line represents delayed sodium group; solid line represents early sodium group. Values are median and interquartile ranges.

tial sodium intake in the first 24 hours from colloid, blood products, medications and flush fluids (median sodium intake ( $\mathrm{mmol} / \mathrm{kg} /$ day), early 3.8; delayed 3.7). There was no difference between the groups in sodium intake during the first 24 hours, but a significant difference up to the time of $6 \%$ weight loss (median sodium intake ( $\mathrm{mmol} / \mathrm{kg} /$ day), early 3.93; delayed $1.07 ; \mathrm{p}=0.0001)$.

\section{RESPIRATORY OUTCOMES}

There was no difference between the groups in the number of babies requiring CPAP or positive pressure ventilation (CPAP/ventilated, early $3 / 21$; delayed $1 / 21$ ), nor in the duration of ventilation (median (range), days, early, $3(0$, $68)$; delayed, $3(0,40))$. By the end of the first week significantly more infants in the delayed group no longer required supplemental oxygen (difference $26 \%, 95 \%$ CI 2, 50). At 28 days after birth and at 36 weeks of postmenstrual age, differences were $22 \%$ (95\% CI -5, 49) and $16 \%(95 \% \mathrm{CI}-14,46)$ (table 2 ).

A regression analysis was performed to evaluate the effect of different variables on duration of additional oxygen treatment. The Cox proportional hazards model incorporated the variables: sodium group, gestational age at

Table 2 Number (\%) of infants in each group no longer requiring supplemental oxygen at 7 and 28 days after delivery and at 36 weeks postmenstrual age

\begin{tabular}{llllll}
\hline & Early & Delayed & $\%$ difference & $95 \%$ CI & p Value \\
\hline 7 days & $2 / 23(9 \%)$ & $7 / 20(35 \%)$ & 26 & 2,50 & 0.03 \\
28 days & $4 / 22(18 \%)$ & $8 / 20(40 \%)$ & 22 & $-5,49$ & 0.12 \\
36 weeks & $11 / 21(52 \%)$ & $13 / 19(68 \%)$ & 16 & $-14,46$ & 0.30 \\
\hline
\end{tabular}

Table 3 Hazard ratios : Cox proportional hazards model

\begin{tabular}{|c|c|c|c|}
\hline & \multicolumn{2}{|c|}{$\begin{array}{l}\text { Hazard ratio } \\
(95 \% \text { confidence interval })\end{array}$} & p Value \\
\hline Birthweight & \multicolumn{2}{|l|}{$16.0(1.5,165)$} & 0.02 \\
\hline Study group & \multicolumn{2}{|l|}{$0.48(0.2,0.98)$} & 0.04 \\
\hline Gender & \multicolumn{2}{|l|}{$0.65(0.31,1.34)$} & 0.24 \\
\hline Gestation & \multirow{2}{*}{\multicolumn{2}{|c|}{$1.09(0.75,1.59)$}} & \multirow{2}{*}{$\begin{array}{l}0.64 \\
0.77\end{array}$} \\
\hline CRIB score & & & \\
\hline \multicolumn{4}{|c|}{$\begin{array}{l}\text { Table } 4 \text { Body weight changes (values are median } \\
\text { (range)) }\end{array}$} \\
\hline & Early & Delayed & $p$ Value \\
\hline $\begin{array}{l}\text { Maximum weight } \\
\text { loss (\%) }\end{array}$ & $\begin{array}{l}11.4 \\
(21.1,2.0)\end{array}$ & $\begin{array}{l}16.0 \\
(22.5,8.5)\end{array}$ & 0.002 \\
\hline $\begin{array}{l}\text { Time to regain birth } \\
\text { weight (days) }\end{array}$ & $12(4,29)$ & $14(10,23)$ & 0.45 \\
\hline $\begin{array}{l}\text { Weight at } 36 \text { weeks } \\
(\mathrm{kg})\end{array}$ & $\begin{array}{l}2.075 \\
(1.335,3.020)\end{array}$ & $\begin{array}{l}2.330 \\
(1.083,2.642)\end{array}$ & NS \\
\hline $\begin{array}{l}\text { Weight at } 6 \text { months } \\
(\mathrm{kg})\end{array}$ & $\begin{array}{l}7.120 \\
(4.450,9.091)\end{array}$ & $\begin{array}{l}6.910 \\
(4.740,8.700)\end{array}$ & NS \\
\hline
\end{tabular}

birth, birthweight, gender and CRIB score. The hazard ratio or risk of no longer requiring additional oxygen was 16 (95\% CI 1.5, 165; $\mathrm{p}=0.02$ ) for birthweight - that is, the heavier the infant at birth, the shorter the requirement for additional oxygen - and 0.48 for sodium group (95\% CI $0.2,0.98 ; \mathrm{p}=0.04)$ - that is, delayed sodium supplementation was associated with a decreased duration of requirement for additional oxygen. Gender, gestational age at birth, and CRIB score were not associated with a significantly altered hazard ratio (table 3 , nor were antenatal administration of thyrotropin releasing hormone, maternal hypertension and symptomatic patent ductus arteriosus.

\section{WEIGHT CHANGES}

There were no significant differences between the two groups in body weight on days 1,7 , or 14 (fig 3). Six babies never achieved $6 \%$ weight loss: one in the delayed group, who died on the sixth day after delivery; and five in the early group, two of whom died in the first week after delivery. In the remaining infants there was no significant difference between the groups in the number of days it took to lose $6 \%$ of birthweight (median (range), early $3(2,7)$; delayed $3.5(2,7)$ ) nor in the number of days it took to regain birthweight (median (range), early 12 (4-29); delayed 14 (10-23)). However, there was a significant difference in maximum weight lost (median (range), early 10.3\% $(0 \%, 21.1 \%)$; delayed $16.1 \%(8.5 \%, 22.5 \%)$, $\mathrm{p}=0.002)$. There were no significant differences between the groups in weight at 36 weeks postmenstrual age (table 4 ).

OTHER CLINICAL OUTCOMES

There was no difference between the groups in serum sodium concentration. During the first three weeks, 12 infants in the early group and 14 infants in the delayed group developed transient hyponatraemia (serum sodium $<130$ $\mathrm{mmol} / \mathrm{l}$ ) and 15 infants in the early group and 11 infants in the delayed group, transient hypernatraemia (serum sodium $>145 \mathrm{mmol} / \mathrm{l}$ ). The numbers of infants in each group (early/delayed) who developed a symptomatic patent ductus arteriosus (9/5), necrotising 
enterocolitis (2/0), intraventricular haemorrhage $(6 / 4)$ or died $(3 / 3)$, were not different between the groups. No infant developed periventricular leucomalacia. One infant in each group developed post-haemorrhagic ventriculomegaly.

\section{Discussion}

Our study set out to address a long standing controversy in neonatal intensive care medicine - namely, the timing of the introduction of routine sodium supplementation. Infants born preterm require sodium supplementation to achieve optimal growth. Sodium is a growth promoter, ${ }^{15-17}$ enhancing growth after the period of supplementation has ceased, ${ }^{18}$ and supplementation is often started routinely after birth. However, given the impaired ability of preterm neonates to excrete a sodium load, we postulated that early sodium supplementation, which would promote persistent expansion of the extracellular compartment and retention of interstitial fluid, might increase respiratory morbidity. The infants in the early group lost significantly less weight than those in the delayed group. As there were no differences in fluid and energy intake between the groups, nor in disease severity, this suggests that the early group had lost less body water.

We aimed to compare outcomes, especially in relation to differences in oxygen dependency and weight gain, in preterm infants receiving sodium supplements from the second day after birth compared with a delay tailored to postnatal weight loss. As the extent of postnatal extracellular fluid loss (and hence postnatal weight loss) is variable, we chose to wait until $6 \%$ weight loss was reached, in view of previous work in which we showed a 5.9\% weight loss after birth in healthy preterm infants. ${ }^{12}$ In our study, this degree of weight loss was reached at around 3-4 days.

To our knowledge, only a single previous study has attempted to address this issue. Costarino et al randomised 17 infants $\leqslant 28$ weeks gestational age to either sodium restriction for five days after birth or sodium supplementation at 3-4 $\mathrm{mmol}$ of sodium $/ \mathrm{kg} /$ day, starting on the second day after birth. ${ }^{19}$ All surviving infants who had received the higher sodium intake developed chronic lung disease, compared with $50 \%$ of survivors from the sodium restricted group. There were no differences between the groups in terms of survival or incidence of patent ductus arteriosus. As in our study, fluid volumes were left to the discretion of the clinicians who were blinded to sodium intake.

The study by Costarino et al was conducted before surfactants and antenatal steroids were in use, whereas in our study almost all infants received antenatal dexamethasone and postnatal surfactant. It is well known that antenatal steroid treatment enhances pulmonary maturity but it is less well appreciated that renal maturation is also accelerated. Glucocorticoids increase the abundance of $\mathrm{Na}^{+}, \mathrm{K}^{+}$-ATPase, the enzyme responsible for sodium transport. ${ }^{20}$ In preterm lambs antenatal betamethasone increases glomerular filtration rate, urine flow, and blood pressure. ${ }^{21}$ In human preterm infants antenatal dexamethasone accelerates maturation of renal function. ${ }^{22} 23$ Antenatal steroid exposure might therefore enhance the ability of newborn preterm infants to excrete a sodium load, reducing the risk of persistent expansion of the extracellular compartment and retention of interstitial fluid. In support of this contention is the observation that both groups of infants lost weight after birth except for a single infant in the delayed sodium group who gained weight after birth and died early in the neonatal period. This infant did not receive steroids antenatally. In the early supplementation group, five infants did not lose $6 \%$ of their birthweight; two of them died in the early neonatal period and one at a few weeks of age. All of these infants had received antenatal dexamethasone. This contrasts with the clinical experience of the era before antenatal steroids were introduced, when failure to lose weight after birth was not uncommon in infants receiving intensive care, and was associated with greater illness severity and poorer outcome. We suggest that antenatal steroid treatment serves to protect preterm neonates from persistent expansion of the extracellular compartment and that this might have reduced differences in outcome between the two groups in our study.

The difference in oxygen dependency between the groups was of similar magnitude at each of three finite time points, one week after birth, 28 days after birth, and 36 weeks of postmenstrual age, namely $26 \%, 22 \%$, and $16 \%$, respectively, although only reaching significance at one week. Our sample size was small and the possibility that this is a type I error cannot be dismissed. Other clinical factors might also have served to reduce differences between the groups. Despite the intention to withhold sodium during the first 24 hours after birth, both groups received a substantial and similar intake of $3.8 \mathrm{mmol} / \mathrm{kg} /$ day in the early group and $3.7 \mathrm{mmol} / \mathrm{kg} /$ day in the delayed group. Significant amounts of unprescribed sodium administration, from medications, flush fluids, and blood products is a well recognised occurrence in neonatal intensive care.

It has been suggested that a high total fluid intake in the first days after birth increases the risk of chronic lung disease. ${ }^{24}$ Unfortunately, few studies have assessed the intake of sodium and water independently. We have already shown that loss of body water after birth occurs to the same extent in healthy preterm neonates and in babies with respiratory distress syndrome and is unrelated to the volume of fluid administered. ${ }^{12}$ Only sodium intake and not total fluid volume was controlled in the present study, but there was no difference between the groups in the total volume of fluid received, nor in the incidence of hypernatraemia or hyponatraemia.

Despite advances in neonatal intensive care, chronic oxygen dependency remains a major problem. We have shown that a small clinical manoeuvre - namely, a delay in routine sodium supplementation - is associated with a significant reduction in the numbers of infants still 
requiring oxygen supplementation at the end of the first week after birth and a decrease in the risk of persisting oxygen requirement. The delay in supplementation did not compromise growth, the incidence of hyponatraemia was not increased, and infants were able to tolerate the same total fluid volume, and therefore receive the same nutritional support, as infants given a higher sodium intake. We therefore recommend a tailored approach to sodium supplementation in preterm infants below 30 weeks of gestation, delaying the start until the postnatal contraction of the extracellular compartment and loss of interstitial fluid is underway. This is likely to be of particular importance in infants who have not been exposed to antenatal glucocorticoid treatment.

PB was supported by a European Union Human Capital and Mobility Grant. We are grateful for help from Ms Caroline Doré, Medical Statistics Unit, Hammersmith Hospital, and Ms Beryl Langfield, Pharmacy, Hammersmith Hospital.

1 Haycock GB. The influence of sodium on growth in infancy Pediatr Nephrol 1993;7:871-5.

2 Shaffer SG, Meade VM. Sodium balance and extracellular volume regulation in very low birth weight infants. F Pediatr 1989;115:285-90.

3 Modi N, Hutton JL. The influence of postnatal respiratory adaptation on sodium handling in preterm neonates. Early Hum Dev 1990;21:11-20.

4 Modi N. Sodium intake and preterm babies. Arch Dis Child 1993;69:87-91.

5 Van Marter LJ, Leviton A, Allred EN, Pagano M, Kuban $\mathrm{KC}$. Hydration during the first days of life and the risk of bronchopulmonary dysplasia in low birth weight infants. $\mathcal{F}$ Pediatr 1990;116:942-9.

6 Stevenson JG. Fluid administration in the association of patent ductus arteriosus complicating respiratory distress syndrome. $\mathcal{F}$ Pediatr 1977;90:257-61

7 Bell EF, Warburton D, Stonestreet BS, Oh W. Effect of fluid administration on the development of symptomatic patent ductus arteriosus and congestive heart failure in premature infants. N Engl f Med 1980;302:598-604.

8 Bell EF, Warburton D, Stonestreet BS, Oh W. High-volume fluid intake predisposes premature infants to necrotising enterocolitis. Lancet 1979;ii:90.

9 Haycock GB, Aperia A. Salt and the newborn kidney. Pediatr Nephrol 1991;5:65-70.
10 Al-Dahan J, Haycock GB, Chantler C, Stimmler L. Sodium homeostasis in term and preterm neonates. II. Gastrointestinal aspects. Arch Dis Child 1983;58:343-5.

11 Speidel B, Fleming PJ, Henderson J, et al, eds. Fluid and electrolyte therapy. In: A Neonatal Vade Mecum. 3rd edn. London: Edward Arnold, 1998: 250-6.

12 Tang W, Ridout D, Modi N. Influence of respiratory distress syndrome on body composition after preterm birth. Arch Dis Child Fetal Neonatal Ed 1997; 77:F28-F31.

13 Al-Rubeyi B, Murray N, Modi N. A variable dextrose delivery system for use in neonatal intensive care. Arch Dis Child Fetal Neonatal Ed 1994;70:F79.

14 International Neonatal Network. The CRIB (clinical risk index for babies) score: a tool for assessing initial neonatal risk and comparing performance of neonatal intensive care units. The International Neonatal Network. Lancet 1993;342:193-8.

15 Chance GW, Radde IC, Willis DM, Roy RN, Park E, Ackerman I. Postnatal growth of infants of less than $1.3 \mathrm{~kg}$ birth weight: effects of metabolic acidosis, of caloric intake, and of calcium, sodium, and phosphate supplementation. $\mathcal{F}$ Pediatr 1977;91:787-93.

16 Wassner SJ. Altered growth and protein turnover in rats fed sodium-deficient diets. Pediatr Res 1989;26:608-13.

17 Ostlund EV, Eklof AC, Aperia A. Salt-deficient diet and early weaning inhibit DNA synthesis in immature rat proximal tubular cells. Pediatr Nephrol 1993;7:41-4.

18 Al-Dahan J, Haycock GB, Nichol B, Chantler C, Stimmler L. Sodium homeostasis in term and preterm neonates. III. Effect of salt supplementation. Arch Dis Child 1984;59:945-50.

19 Costarino AT, Gruskay JA, Corcoran L, Polin RA, Baumgart S. Sodium restriction versus daily maintenance replacement in very low birth weight premature neonates: a randomized, blind therapeutic trial. I Pediatr 1992;120:99-106.

20 Celsi G, Nishi A, Akusjarvi G, Aperia A. Abundance of $\mathrm{Na}(+)-\mathrm{K}(+)$-ATPase mRNA is regulated by glucocorticoid hormones in infant rat kidneys. Am $\mathcal{F}$ Physiol 1991;260:F192-7.

21 Ervin MG, Berry LM, Ikegami M, Jobe AH, Padbury JF, Polk DH. Single dose fetal betamethasone administration stabilizes postnatal glomerular filtration rate and alters endocrine function in premature lambs. Pediatr Res 1996;40:645-51.

22 Al-Dahan J, Stimmler L, Chantler C, Haycock GB. The effect of antenatal dexamethasone administration on glomerular filtration rate and renal sodium excretion in premature infants. Pediatr Nephrol 1987;1:131-5.

23 Zanardo V, Giacobbo F, Zambon P, et al. Antenatal aminophylline and steroid exposure: effects on glomerular filtration rate and renal sodium excretion in preterm newborns. f Perinat Med 1990;18:283-8.

24 Bell EF. Fluid therapy. In: Sinclair JC, Bracken MB, eds. Effective care of the newborn. Oxford: Oxford University Press, 1992:59-71. 